\title{
Tumores ováricos: correlación entre los informes de estudios solicitados por congelación y la histopatología definitiva. Hospital Universitario del Valle 1994 - 1997
}

\author{
José Saulo Torres R, MD*; Juan Pablo Suso, MD**; Eduardo Perea, MD**; Luis Alberto Tafur C., MD***; \\ Marisol Agudelo, $\mathrm{MD}^{* * * *}$
}

\begin{abstract}
RESUMEN:
Se analizó la sensibilidad y la especificidad de las biopsias por congelación versus informe definitivo en el Hospital Universitario del Valle, adicionalmente se enfatiza sobre los beneficios de este proceder.
\end{abstract}

PALABRAS CLAVES: Ovario, Congelación, Informe definitivo, Mala práctica.

SUMMARY:

Sensitivity and specificity of frozen biopses were analyzed against a final report at Valle University Hospital; additionally benefits from this procedure are emphasized.

KEY WORDS: Ovary, Freezing, Final report, Poor practice.

Ante la sospecha clínica de tumor ovárico se recomiendan exámenes previos a la laparotomía llamrados de extensión (1). Ninguno de estos exámenes es suficientemente sensible para determinar el comportamiento biológico del tumor (2), sólo se tiene una aproximación diagnóstica adecuada a través de la biopsia por congelación, la cual se constituye en la piedra angular para tomar cualquier decisión razonable en beneficio de la paciente, que en grupos bien experimentados puede llegar a una sensibilidad del 93\%(3). La biopsia por congelación es un procedimiento ágil Para establecer el carácter benigno o maligno y tipo histológico de la lesión y orienta al cirujano en la radicalidad del procedimiento quirúrgico. El presente estudio permite comparar los resultados de la biopsia por congelación con el estudio histopatologico final de los tumores ováricos operados en el H.U.V. de Cali entre enero de 1994 y diciembre de 1997 con el propósito de determinar la utilidad y limitaciones del método.

Se operaron 199 pacientes con tumores ováricos en el Hospital en ese período, de los cuales 126 se les realizó biopsia por congelación.

Jefe sección Ginecología Oncológica H.U.V. docente Ad-honorem Universidad del Valle Cali Colombia

Ginecólogos Oncólogos H.U.V. Profesores Ad-honorem Universidad del Valle

Especialista Salud Pública. Profesor Titular Escuela de Salud Pública Universidad del Valle

Residente de tercer año Universidad Pontificia Bolivariana Medellín Colombia.

\section{Materiales y métodos}

Se compararon los informes de las biopsias por congelación de los especímenes con el diagnóstico histopatológico definitivo.

De los 199 tumores de ovario operados en dicho periodo el diagnóstico final fue: Benignos: 109 (55\%), B.P.M: 15 (7\%), Malignos: 75 (38\%), de estos el $63.4 \%$ tenia estudio de congelación. Los casos en que se omitió la biopsia por congelación (73 casos, 36.6\%) corresponde: a) Daño del equipo. B) Evidencia clínica de malignidad a criterio del cirujano aunado a la edad y paridad completa de la paciente. C) Pacientes que se intentaron operar en otros centros y fueron remitidas sólo con el diagnóstico histológico por considerarse inoperables.

El diagnóstico por congelación se clasificó en cuatro categorías: Benigno, Bajo Potencial de Malignidad (BPM: Son tumores epiteliales con una baja tasa de crecimiento y un bajo potencial para invadir o metastatizar 3), Malignos y 4) Diferidos (dificultad del patólogo de emitir un concepto de malignidad, frente al espécimen examinado). La información se recogió de los archivos de historias clínicas de la sección de Ginecología Oncológica del H.U.V

Se calculó el coeficiente de correlación Kappa entre las dos lecturas, la sensibilidad, especificidad, valor predictivo positivo y negativo de la biopsia por congelación comparativamente con el resultado histopatológico. 
Tabla 1

COMPARACION DEL DIAGNOSTICO HISTOLOGICO DEFINITIVO Y EL DIAGNOSTICO POR BIOPSIA DE CONGELACION. H.U.V.

\begin{tabular}{|c|c|c|c|c|}
\hline DX DEFINITIVO & BENIGNO & B.P.M & MALIGNO & TOTAL \\
\hline $\begin{array}{c}\text { DX POR } \\
\text { CONGELACION }\end{array}$ & & & & \\
\hline BENIGNO & 78 & 8 & 6 & 92 \\
\hline B.P.M & 0 & 0 & 1 & 1 \\
\hline MALIGNO & 0 & 2 & 28 & 30 \\
\hline DIFERIDO & 1 & 1 & 1 & 3 \\
\hline TOTAL & $\mathbf{7 9}$ & $\mathbf{1 1}$ & $\mathbf{3 6}$ & $\mathbf{1 2 6}$ \\
\hline
\end{tabular}

Tabla 2

RESULTADOS DE LAS PRUEBAS ESTADISTICAS DE COMPARACION ENTRE BIOPSIA POR CONGELACION E HISTOPATOLOGIA FINAL

\begin{tabular}{|l|l|c|c|}
\hline \multicolumn{1}{|c|}{ PRUEBA } & DIAGNOSTICO & VALOR & \multicolumn{1}{c|}{ IC } \\
\hline KAPPA & BENIGNO & 0.73 .385 & $\begin{array}{c}\mathrm{Z}: 8.43 \\
\mathrm{P}: 0.000000\end{array}$ \\
\cline { 2 - 4 } & MALIGNO & 0.795322 & $\begin{array}{c}\mathrm{Z}: 9.0 \\
\mathrm{P}: 0.000000\end{array}$ \\
\hline \multirow{3}{*}{ SENSIBILIDAD } & BENIGNO & 98.7 & $\mathrm{IC}: 92.2-99.9$ \\
\cline { 2 - 4 } & BPM & 0 & $\cdot$ \\
\cline { 2 - 4 } & MALIGNO & 77.8 & $60.4-89.3$ \\
\hline ESPECIFICIDAD & BENIGNO & 70.2 & $54.9-82.2$ \\
\cline { 2 - 4 } & MALIGNO & 86.7 & $77.5-92.6$ \\
\hline \multirow{2}{*}{ V. P.(+) } & BENIGNO & 84.8 & $75.4-91.1$ \\
\cline { 2 - 4 } & MALIGNO & 70.0 & $53.3-82.9$ \\
\hline \multirow{2}{*}{ V. P. (-) } & BENIGNO & 97.1 & $82.9-99.8$ \\
\cline { 2 - 4 } & MALIGNO & 90.7 & $82.0-95.6$ \\
\hline
\end{tabular}

Tabla 3

\section{SENSIBILIDAD EN LA BIOPSIA POR CONGELACIÓN EN TUMORES OVÁRICOS EN DISTINTOS ESTUDIOS. (2)}

\begin{tabular}{|l|c|c|c|c|}
\hline \multicolumn{1}{|c|}{ Autor } & No. & $\begin{array}{c}\text { Benignos } \\
(\mathbf{\%})\end{array}$ & $\begin{array}{c}\text { Maligno } \\
(\mathbf{\%})\end{array}$ & $\begin{array}{c}\text { B.P.M. } \\
(\mathbf{\%})\end{array}$ \\
\hline Slavutin and Rotterdam & 55 & 97.1 & 73.7 & 50.0 \\
\hline Bastos de Cunha y cols. & 120 & 97.5 & 89.7 & 0.0 \\
\hline Twaalfhoven y cols. & 176 & 92.8 & 88.5 & 44.4 \\
\hline Obiakor y cols. & 303 & 100.0 & 93.2 & 0.0 \\
\hline Rose PG. y Cols. & 383 & 98.7 & 92.5 & 44.8 \\
\hline Torres JS y Cols. & 126 & 98.7 & 77.8 & 0.0 \\
\hline
\end{tabular}

\section{Resultados}

126 tenían informe por congelación así:

$\begin{array}{lrr}\text { Benignos } & 92 & (73.0 \%) \\ \text { B.P.M. } & 1 & (0.8 \%) \\ \text { Malignos } & 30 & (24.0 \%) \\ \text { Diferidos } & 3 & (2.2 \%)\end{array}$

En la tabla 1 se presenta en forma comparativa los resultados de ambas lecturas: congelación vs informe definitivo.

En la tabla 2 se encuentran los resultados de las pruebas estadísticas Kappa, sensibilidad, especificidad y valor predictivo positivo y negativo.

Entre los 8 casos con diagnóstico incorrecto en el reporte de congelación un tumor era de B.P.M. 6 benig- nos y uno diferido. Se observo por ejemplo como dos tumores de la granulosa fueron interpretados como disgerminomas. Tres tumores $(8.3 \%)$ correspondieron a tumores metastásicos.

\section{Discusión}

La sección de Oncología Ginecológica del Hospital Universitario del Valle Evaristo García realiza desde hace 5 años en forma protocolario biopsias por congelación de los especímenes de ovario.

En el presente estudio la sensibilidad (posibilidad de clasificar como positivo a un verdadero positivo) de los reportes de congelación para tumores benignos y malignos fue de $98.7 \%$ y $77.8 \%$ respectivamente. Esto se compara en la tabla 3 con otros reportes de diagnósticos de cortes por congelación de tumores de Ovario (3).

Los informes que presentaron disparidad entre el informe definitivo y la biopsia inicial por congelación se comentan a continuación: De los 92 tumores reportados como Benignos existió acuerdo en 78 siendo 14 los dispares. De estos 6 fueron malignos, errores clínicamente importantes en él diagnostico por congelación. Otros 8 eran tumores de bajo potencial de malignidad y 5 coincidieron como benignos pero el tipo histológico estuvo equivocado.

La sensibilidad para tumores benignos fue del $98.73 \%$, la cual se compara favorablemente con el $92.8 \%$ a $100 \%$ de sensibilidad de otros reportes. Lo más importante para este tipo de tumores es la especificidad (posibilidad de clasificar como negativo para malignidad un tumor benigno) la cual fue del $70.2 \%$ y se incrementa al $92.93 \%$ si se excluían los tumores de bajo potencial de malignidad.

Respecto a los tumores de Bajo Potencial De Malignidad se reportó sólo uno en esta serie, no concidiendo el grado de malignidad, ni el subgrupo histologico. Este diagnóstico requiere una sección histológica por $\mathrm{cm}$ del diámetro mayor del tumor, lo cual es difícil para los patólogos quienes están presionados por el tiempo, son tumores voluminosos generalmente y de apariencia benigna. Estudios previos han mostrado una sensibilidad baja comprendida entre el 0 y el $50 \%$. El diagnóstico intraoperatorio de un tumor de bajo potencial de malignidad no es tan importante debido a su comportamiento biológico el cual es semejante al de los tumores benignos y no se ha establecido quimioterapia complementaria (5). Nuestra sensibilidad fue de $0 \%$.

En cuanto a los tumores Malignos se identificaron correctamente el $77.8 \%$ de los casos. En otros estudios, la sensibilidad ha variado de $73.7 \%$ a $93.2 \%$. Entre los 8 pacientes incorrectamente diagnosticados, 6 lo fueron como benignos, uno como B.P.M. y uno Diferido. Tumores de gran tamaño con un pequeño foco de tumor maligno pueden pasar de alta en la congelación y evidenciarse en los estudios de parafina. La especificidad para estos tumores fue de $94.7 \%$.

Como diferidos solo se reportaron 3 casos de los 126 . $\mathrm{El}$ análisis de los diagnósticos definitivos muestra que no era fácil a la congelación para los examinadores comprometerse con un diagnostico, por fortuna para los cirujanos esto se dio en un mínimo numero de pacientes $(1.5 \%$.). 
Comparación entre diagnóstico Diferido y Diagnóstico histopatológico definitivo.

\begin{tabular}{|l|l|l|c|l|}
\hline (1) & $D$ & Diferido & $\begin{array}{c}\text { BP } \\
M\end{array}$ & $\begin{array}{l}\text { Tumor seroso papilar de malignidad } \\
\text { intermedia }\end{array}$ \\
\hline (2) & $D$ & Diferido & $M$ & Adenocarcinoma de células claras \\
\hline (3) & $D$ & Tumor Sólido no mas datos & $B$ & Fibrotecoma Ovario Derecho \\
\hline
\end{tabular}

En nuestro estudio, los resultados indican un buen acuerdo entre las lecturas de la biopsia por congelación y la histopatología definitiva definida por el resultado de la prueba Kappa, la cual mide una prueba de tamiz contra la considerada de oro, indicando la calidad de los patólogos que las realizan.

Entre los factores posibles de no acuerdo, se encuentran: a) Lecturas por observadores diferentes con distinto nivel de capacitación b) Las características propias del tumor, ya comentadas; c) La necesidad de producir un resultado en poco tiempo en el caso de la congelación, presión que puede llevar a leer rápida e inadecuadamente un espécimen.

Al comparar con estudios similares se observa que la sensibilidad de la lectura de tumores benignos es la informada por ellos, mientras que la de los malignos está en el rango inferior de los valores reportados.

\section{Resumen}

La biopsia por congelación es un procedimiento ágil para establecer el carácter benigno o maligno y tipo histológico de la lesión y orienta al cirujano en la radicalidad del procedimiento quirúrgico. Con el objetivo de comparar los resultados de la biopsia por congelación con el estudio histopatológico final de los tumores ováricos operados en el HUV de Cali entre Enero de 1994 y Diciembre de 1997, se compararon los informes de 126 biopsias por congelación y del resultado histopatológico final de 199 tumores ováricos operados en el HUV en ese período. A 73 no se les realizó por: a) Evidencia macroscopica de malignidad b) Por tener resultado histopatológico definido a consecuencia de laparatomía incompleta donde solo se tomo la biopsia y se cerró de nuevo el paciente por considerarlo inoperable, c) Por daño del equipo en el departamento de patología.

Con base en la comparación se calcularon la sensibilidad, especificidad y valores predictivos, así como el coeficiente de correlación existente entre las dos lecturas. De los 199 casos recopilados se descartaron 73 (36.6\%) por carecer del informe de la biopsia por congelación.

El diagnóstico por congelación realizado en 126 pacientes estuvo de acuerdo con el histopatológico en el $77 \%$ de los casos. La sensibilidad para tumores malignos fue $77.8 \%$ (IC: 60.4 - 89.3) para BPM $0 \%$ y benignos 98.7\% (LC: 92.2 - 99.9). La especificidad para tumores benignos fue del 70.2 (LC:54.9 - 82.2) pero se incrementa al $93 \%$ si los tumores de BPM eran excluidos. El coeficiente Kappa para benignidad entre las dos pruebas fue de 0.73 y para malignidad de 0.79 lo cual indica un buen acuerdo entre las dos informes.

\section{Conclusiones}

1) La interconsulta al departamento de Patología para confirmar la malignidad o benignidad de la pieza tumoral y su tipo histológico es de fundamental importancia, y debe ser un requisito indispensable para los centros donde se realizan este tipo de cirugía, pues hasta no conocer el grado de malignidad y su tipo histológico no se debe realizar la cirugía adecuada teniendo implicaciones tanto intraoperatorias, postoperatorias como legales.

2) La idoneidad y dedicación del grupo humano que realiza el examen de la pieza remitida para su diagnóstico por congelación es de importancia fundamental así como la del grupo quirúrgico en la interpretación del diagnóstico y el conocimiento del comportamiento biológico de la enfermedad, en especial cuando se relaciona con el manejo y preservación de la fertilidad.

3) El acuerdo observado entre la biopsia de congelación y la histopatología en el HUV índica una buena correlación entre los dos informes.

\section{BIBLIOGRAFIA}

1. Disaia-Creasman. Oncología Ginecológica Clínica. Estadiaje. Pag:675-81.Mosby/Doyma 1994. España.

2. González Bárón M. Oncología Clínica. Tumores de Ovario pag: 207. Interamericana-McGraw-Hill. 1992 España.

3. Rose PG, Rubin RB, Nelson BE, Hunter RE y Reale FR.Accuracy of frozen-section (intraoperative consultation) diagnosis of ovarian tumors. Am J Obstet Ginecol 1994; 171: 823-6
4. Charles J Link Jr, Eddie Reed, Gisele Sarosy, Elise C Kohn. Borderline Ovarian Tumors, Review. The American Journal of Medicine. Volume 101. Agost 1996.Pag: 217-25.

5. Tazelaar H.D., Bostwick DG, Ballon SC, Hendrickson MR, Kempson RL.Conservative treatment of borderline Ovarian Tumors. Obst Gynecol 1985,66:417-22. 